\title{
The Super-Poincaré Algebra Via Pure Spinors and the Interaction Principle in 3D Euclidean Space
}

\author{
R. da Rocha \\ IFGW, Universidade Estadual de Campinas, CP 6165, 13083-970 Campinas, SP, Brazil
}

(Received on 12 September, 2005)

\begin{abstract}
The Poincare superalgebra is introduced from a generalization of the Cartan's triality principle based on the extension of Chevalley product, between semispinor spaces and even subspaces of the extended exterior algebra over Euclidean space $\mathbb{R}^{3}$. The pure spinor formalism and the framework of Clifford algebras are used, in order to provide the necessary tools to introduce the Poincare superalgebra where all the operators in space and superspace are constructed via pure spinors in $\mathbb{R}^{3}$ and the interaction principle, that generalizes the $\mathrm{SO}(8)$ triality principle.
\end{abstract}

\section{INTRODUCTION}

The seminal paper of Wess and Zumino [1] treating the 4dimensional super-Poincaré algebra, has boosted new investigations on supersymmetry in Lagrangian field theory and in particle physics. On the other hand, Cartan's triality principle, based on the group $\mathrm{SO}(8)$ and its double covering Spin(8), has been applied in recent physical theories, like supergravity and superstrings. Only in dimension 8, bosons and fermions have the same number of degrees of freedom. This generalized supersymmetry comes from the triality principle, which asserts that bosons and fermions are equivalent under the triality map [2-4], based on the Chevalley product [4]. This paper is presented as follows: in Section 2 the main results involving the structure of the Chevalley product, giving rise to the triality principle, are presented [2], and we briefly revisit the pure spinor formalism. In Section 3 the Poincaré superalgebra is introduced in $\mathbb{R}^{3}$, via orthosymplectic graded Lie algebras and via the interaction principle, which generalizes the cyclic property of the Chevalley product. This work was supported by CAPES.

\section{TRIALITY PRINCIPLE AND PURE SPINORS}

Consider a vector space $V$ endowed with metric $g$. The spinor space $\mathbf{S}$ can be written as the direct sum $\mathbf{S}=\mathbf{S}^{+} \oplus \mathbf{S}^{-}$, where $\mathbf{S}^{ \pm}$denotes semispinor spaces that carry, each one, a non-equivalent irreducible representation of the even subalgebra $C \ell^{+}(V, g)$. Define the space $\mathbf{E}=V \oplus \mathbf{S}^{+} \oplus \mathbf{S}^{-}$. In order to find a vector space $V$ such that the semispinor space $\mathbf{S}^{ \pm}$of $V$ has the same dimension $n$ of $V$, the condition $n=8$ must hold. We consider hereon $V \simeq \mathbb{R}^{8,0}$ [5]. The space $\mathbf{E}$ is therefore 24-dimensional and its elements are hereon denoted by $\phi=\mathbf{x}+\mathbf{u}+\mathbf{v}$, where $\mathbf{x} \in V, \mathbf{u} \in \mathbf{S}^{+}$and $\mathbf{v} \in \mathbf{S}^{-}$. The spinor metric $h: \mathbf{S}^{ \pm} \times \mathbf{S}^{ \pm} \rightarrow \mathbb{R}$ is defined as

$$
h(\mathbf{u}, \mathbf{x v})=h(\mathbf{x u}, \mathbf{v})=(\mathbf{x u})^{\chi} \mathbf{v}
$$

where $(\cdot)^{\chi}$ is a dual operator from $\mathbf{S}^{ \pm}$to $\left(\mathbf{S}^{ \pm}\right)^{*}$. A metric $\breve{B}: \mathbf{E} \times \mathbf{E} \rightarrow \mathbb{R}$ is defined from the spinor metric $h$ and the metric $g$ :

$$
\breve{B}\left(\phi_{1}, \phi_{2}\right)=g\left(\mathbf{x}_{1}, \mathbf{x}_{2}\right)+h\left(\mathbf{u}_{1}, \mathbf{u}_{2}\right)+h\left(\mathbf{v}_{1}, \mathbf{v}_{2}\right) .
$$

A totally symmetric trilinear tensor is defined as

$$
\begin{aligned}
T\left(\phi_{1}, \phi_{2}, \phi_{3}\right) & =h\left(\mathbf{u}_{1}, \mathbf{x}_{2} \mathbf{v}_{3}\right)+h\left(\mathbf{u}_{1}, \mathbf{x}_{3} \mathbf{v}_{2}\right)+h\left(\mathbf{u}_{2}, \mathbf{x}_{3} \mathbf{v}_{1}\right) \\
& +h\left(\mathbf{u}_{2}, \mathbf{x}_{1} \mathbf{v}_{3}\right)+h\left(\mathbf{u}_{3}, \mathbf{x}_{2} \mathbf{v}_{1}\right)+h\left(\mathbf{u}_{3}, \mathbf{x}_{1} \mathbf{v}_{2}\right) .
\end{aligned}
$$

It is possible to endow the vector space $\mathbf{E}$ with an associative and non-commutative product $\triangle: \mathbf{E} \times \mathbf{E} \rightarrow \mathbf{E}$, called the Chevalley product, that is implicitly defined by $[2,4]$ $T\left(\phi_{1}, \phi_{2}, \phi_{3}\right)=\breve{B}\left(\phi_{1} \triangle \phi_{2}, \phi_{3}\right)$. It is immediate to see that $\mathbf{x} \triangle \mathbf{u}=\mathbf{x u}$ and $\mathbf{x} \triangle \mathbf{v}=\mathbf{x v}$ [2].

- Proposition 1: The inclusions $V \triangle \mathbf{S}^{+} \subseteq \mathbf{S}^{-}, \quad \mathbf{S}^{+} \triangle$ $\mathbf{S}^{-} \subseteq V, \quad \mathbf{S}^{-} \triangle V \subseteq \mathbf{S}^{+}$hold.

Hereon $\mathbf{x}, \mathbf{u}$ and $\mathbf{v}$ are respectively denoted elements of $V, \mathbf{S}^{+}$and $\mathbf{S}^{-}$. The spinor representation $\rho$ of the Clifford product in $\mathbf{S}^{ \pm}$and the vector (adjoint) representation $\chi$ in $V$ naturally induce a irreducible representation $Y$ in $\mathbf{E}$. Given $s \in \Gamma^{+}$, that denotes the Clifford group of elements of unit norm, such representation is defined by $Y(s)(\mathbf{x}+\mathbf{u}+\mathbf{v})=$ $\chi(s) \mathbf{x}+\rho(s) \mathbf{u}+\rho(s) \mathbf{v}$.

The representation $Y(s)$ is shown to be orthogonal in relation to the metric on $\breve{B}$ [2]. In addition, it can be shown that, if $\mathbf{x}_{0} \in \mathbf{V}$ is such that $\mathbf{x}_{0}^{2}=1$, then $Y^{2}\left(\mathbf{x}_{0}\right)=1$. Now consider a spinor $\mathbf{u}_{0} \in \mathbf{S}^{+}$of unitary norm, i.e., $h\left(\mathbf{u}_{0}, \mathbf{u}_{0}\right)=1$. A homomorphism $\tau: \mathbf{S}^{+} \rightarrow V$ is defined is such a way that $\tau\left(\mathbf{u}_{0}\right)(\mathbf{x})=$ $\mathbf{x} \triangle \mathbf{u}_{0}$. The map $\tau\left(\mathbf{u}_{0}\right)$ is clearly orthogonal and is uniquely extended to an order two automorphism in $V \oplus \mathbf{S}^{-}$. If $\mathbf{v} \in \mathbf{S}^{-}$is such that $\mathbf{v}=\tau\left(\mathbf{u}_{0}\right) \mathbf{x}$ for a unique $\mathbf{x} \in V$, defining $\tau\left(\mathbf{u}_{0}\right)(\mathbf{v})=\mathbf{x}$, and besides, the map $\tau\left(\mathbf{u}_{0}\right)$ is defined in $\mathbf{S}^{+}$as a reflection, given a fixed spinor $\mathbf{u}_{0} \in \mathbf{S}^{+}$, as $\tau\left(\mathbf{u}_{0}\right)(\mathbf{u})=2 h\left(\mathbf{u}, \mathbf{u}_{0}\right) \mathbf{u}_{0}-\mathbf{u}$. In order to define an order-three automorphism, that permutes cyclically the vector spaces $\mathbf{S}^{+}, \mathbf{S}^{-}$and $V$, we can verify that $\tau\left(\mathbf{u}_{0}\right) Y\left(\mathbf{x}_{0}\right) \tau\left(\mathbf{u}_{0}\right)=Y\left(\mathbf{x}_{0}\right) \tau\left(\mathbf{u}_{0}\right) Y\left(\mathbf{x}_{0}\right)$, and then the triality operator $\Theta$ is defined as $\Theta\left(\mathbf{x}_{0}, \mathbf{u}_{0}\right)=Y\left(\mathbf{x}_{0}\right) \tau\left(\mathbf{u}_{0}\right)$, which is an order three automorphism, since $\Theta^{3}\left(\mathbf{x}_{0}, \mathbf{u}_{0}\right)=1$.

- Proposition 2: The subspaces $V, \mathbf{S}^{+}$and $\mathbf{S}^{-}$of $\mathbf{E}$ are cyclically permuted by $\Theta\left(\mathbf{x}_{0}, \mathbf{u}_{0}\right)$, in such way that $\Theta\left(\mathbf{x}_{0}, \mathbf{u}_{0}\right) . V \subset$ $\mathbf{S}^{+}, \quad \Theta\left(\mathbf{x}_{0}, \mathbf{u}_{0}\right) . \mathbf{S}^{+} \subset \mathbf{S}^{-}, \quad \Theta\left(\mathbf{x}_{0}, \mathbf{u}_{0}\right) . \mathbf{S}^{-} \subset V$.

Now pure spinor formalism is briefly reviewed. For more details, see e.g. [2-4, 6-8]. Given the complex $\mathbb{C}^{2 n}$ vector space and its corresponding Clifford algebra $C \ell(2 n, \mathbb{C})$ with generators $\mathbf{x}^{a}$, a spinor $\mathfrak{u}$ is a vector of the $2 n$-dimensional representation space of $C \ell(2 n, \mathbb{C})=$ End $\mathbf{S}$, defined by the Cartan's equation $z \mathfrak{u}=0$. For $\mathfrak{u} \neq 0, z \in \mathbb{C}^{2 n}$ may only be null. Defining the volume element $z$, Weyl spinors $\mathfrak{u}_{ \pm}$are defined 
by $z\left(1 \pm \mathbf{x}_{2 n+1}\right) \mathfrak{u}_{ \pm}=0$, and are elements of the representation space of the even subalgebra $C \ell^{+}(2 n, \mathbb{C})$. Each Weyl spinor $\mathfrak{u}_{ \pm}$spans a $2^{n-1}$-dimensional spinor space, and the expression $z\left(1 \pm \mathbf{x}_{2 n+1}\right) \mathfrak{u}_{ \pm}=0$ defines $d$-dimensional totally null planes $T_{d}\left(\mathfrak{u}_{ \pm}\right)$. It may be shown that every $\mathfrak{u}_{ \pm} \in \mathbf{S}^{ \pm}$, where $C \ell^{+}(2 n, \mathbb{C})=$ End $\mathbf{S}^{ \pm}$define such planes. For $d=n$, that is, for maximal dimension of the totally null planes $T_{n}\left(\mathfrak{u}_{ \pm}\right):=$ $M\left(\mathfrak{u}_{ \pm}\right)$, the corresponding Weyl spinor $\mathfrak{u}_{ \pm}$is named simple or pure and, as shown by Cartan, $M\left(\mathfrak{u}_{ \pm}\right)$and $\pm \mathfrak{u}_{ \pm}$are isomorphic $\left(\bmod \mathbb{Z}_{2}\right)$. All Weyl spinors are simple or pure for $n \leq 3$.

\section{POINCARÉ SUPERALGEBRA IN $\mathbb{R}^{3}$}

In four dimensions the (translational) super-Poincaré algebra [1] is given by

$$
\begin{aligned}
& \left\{Q_{a}, Q_{b}\right\}=2 \sigma_{a \dot{b}}^{\mu} P_{\mu}, \quad\left[P_{\mu}, P_{v}\right]=0 \\
& \left\{Q_{a}, Q_{b}\right\}=\left\{Q_{\dot{a}}, Q_{\dot{b}}\right\}=\left[P_{\mu}, Q_{a}\right]=\left[P_{v}, Q_{\dot{b}}\right]=0
\end{aligned}
$$

The operators $P_{\mu}, Q_{a}$ can be written, in terms of the superspace coordinates, as $P_{\mu}=i \partial_{\mu}$, and

$$
Q_{a}=\partial_{\theta^{a}}-i \sigma_{a \dot{b}}^{\mu} \theta^{\dot{b}} \partial_{\mu}, \quad Q_{\dot{a}}=-\partial_{\theta^{\dot{a}}}+i \theta^{b} \sigma_{b \dot{a}}^{\mu} \partial_{\mu}
$$

We now extend the results in [6], where the Poincaré superalgebra is extended by generalizing Prop.1. Instead of considering a vector space $V$, it is possible to consider any subspace $\mathcal{A}$ of the Clifford algebra $C \ell(V, g)$. Denoting $\mathcal{A}^{ \pm} \subseteq$ $C \ell^{ \pm}(V, g)$, where $C \ell^{-}(V, g)$ denotes the subspace of multivectors of $C \ell(V, g)$ written as the Clifford product of an odd number of vectors in $V$, the proposed generalization in [6] allows two possibilities: a) If $\operatorname{dim}(V)=n$ is such that $n / 2$ is odd, we have: $\left.\mathcal{A}^{+} \triangle \mathbf{S}^{+} \subseteq \mathbf{S}^{+} \quad \mathbf{S}^{+} \Delta \mathbf{S}^{-} \subseteq \mathcal{A}^{+}, \quad \mathbf{S}^{-} \triangle \mathcal{A}^{+} \subseteq \mathbf{S}^{-} ; \mathrm{b}\right)$ If $\operatorname{dim}(V)=n$ is such that $n / 2$ is even, it follows that $\mathcal{A}^{-} \Delta \mathbf{S}^{+} \subseteq \mathbf{S}^{-} \quad \mathbf{S}^{+} \Delta \mathbf{S}^{-} \subseteq \mathcal{A}^{-}, \quad \mathbf{S}^{-} \triangle \mathcal{A}^{-} \subseteq \mathbf{S}^{+}$. This is the so-called interaction principle [6]. Taking a basis of $\left(\mathbb{R}^{3} \oplus \mathbb{R}^{3}\right)$, as being $\left\{\mathbf{e}_{j}, \stackrel{\circ}{j}_{j}\right\}_{j=1}^{3}$, define the vectors $\xi_{i}$ as

$$
\begin{aligned}
& 2 \xi_{0}=\mathbf{e}_{1}+\stackrel{\circ}{3}_{3}, \quad 2 \xi_{1}=\mathbf{e}_{2}+\stackrel{\mathbf{e}}{1}_{1}, \quad 2 \xi_{2}=i \mathbf{e}_{3}+\stackrel{\mathbf{e}}{1}_{1}, \\
& 2 \xi_{3}=\mathbf{e}_{1}-\stackrel{\circ}{3}_{3}, \quad 2 \xi_{4}=\mathbf{e}_{2}-\check{\mathbf{e}}_{1}, \quad 2 \xi_{5}=i \mathbf{e}_{3}-\check{\mathbf{e}}_{1}
\end{aligned}
$$

where $2 g\left(\xi_{i}, \xi_{3+i}\right)=1=\xi_{i} \xi_{3+i}+\xi_{3+i} \xi_{i}, \quad 0 \leq i \leq 2$ [6]. Choosing the subspace of the exterior algebra, the underlying vector space of the Clifford algebra, as

$$
\mathcal{A}=\Lambda_{\mathbb{C}}^{2}\left(\mathbb{R}^{3} \oplus \mathbb{R}^{3}\right) \oplus \Lambda_{\mathbb{C}}^{6}\left(\mathbb{R}^{3} \oplus \mathbb{R}^{3}\right),
$$

in particular the following products can be obtained:

$$
\begin{aligned}
\xi_{3} \xi_{4} \xi_{5} \Delta \xi_{1} \xi_{4} & =2 \xi_{5} \xi_{3}, \xi_{3} \xi_{4} \xi_{5} \Delta \xi_{2} \xi_{3} \xi_{5}=-2 \xi_{5} \xi_{3}, \\
\xi_{1} \xi_{2} \xi_{3} \Delta \xi_{1} \xi_{4} \xi_{5} & =2 \xi_{1} \xi_{3}, \xi_{1} \xi_{2} \xi_{3} \Delta \xi_{3} \xi_{4}=2 \xi_{2} \xi_{3} .
\end{aligned}
$$

From the properties $\left\{\mathfrak{v} \xi_{3} \xi_{4} \xi_{5}, \mathfrak{w} \xi_{3} \xi_{4} \xi_{5}\right\}=-\left(\mathfrak{v} \xi_{3} \xi_{4} \xi_{5}\right) \Delta$ $\left(\mathfrak{w} \xi_{3} \xi_{4} \xi_{5}\right),\left\{\mathfrak{v} \xi_{3} \xi_{4} \xi_{5}, \mathfrak{w} \xi_{3} \xi_{4} \xi_{5}\right\}=\left\{\mathfrak{v} \xi_{3} \xi_{4} \xi_{5}, \mathfrak{w} \xi_{3} \xi_{4} \xi_{5}\right\}=$ $0,[\psi, \phi]=0$, where $\mathfrak{v}, \mathfrak{w} \in \mathcal{A}^{ \pm}, \psi, \phi=\xi_{A} \mathbf{e}_{i}$ or $\xi_{A} \check{\mathbf{e}}_{i}$, and using the results in [6], if we define

\begin{tabular}{||r||r|r|r||}
\hline & $Q_{\mu}$ & $Q_{\dot{\mu}}$ & $P_{\mu}$ \\
\hline \hline$\mu=0$ & $\xi_{1} \xi_{4} \xi_{3}+\xi_{0} \xi_{3} \xi_{4}$ & $\xi_{0} \xi_{2} \xi_{4} \xi_{5}+\xi_{3} \xi_{4} \xi_{5}$ & $\xi_{2} \xi_{4} \xi_{5}+\xi_{0} \xi_{1} \Delta \xi_{2} \xi_{3}$ \\
\hline$\mu=1$ & $\xi_{2} \xi_{4} \xi_{5}-\xi_{1} \xi_{3} \xi_{5}$ & $-\xi_{2} \xi_{3} \xi_{4}-\xi_{1} \xi_{2} \xi_{5}$ & $\xi_{1} \xi_{0} \xi_{5}+\xi_{3} \xi_{4} \Delta \xi_{1} \xi_{2} \xi_{5}$ \\
\hline$\mu=2$ & $\xi_{3} \xi_{4} \xi_{5}+\xi_{1} \xi_{3} \xi_{4}$ & $-\xi_{3} \xi_{4} \xi_{5}+\xi_{1} \xi_{2}$ & $\xi_{3} \xi_{4} \Delta \xi_{1} \xi_{3} \xi_{4} \xi_{5}-\xi_{4}$ \\
\hline$\mu=3$ & $\xi_{0} \xi_{4} \xi_{5}-\xi_{3} \xi_{4} \xi_{5}$ & $\xi_{3} \xi_{4} \xi_{5}+\xi_{0} \xi_{2} \xi_{5}$ & $\xi_{2} \xi_{3} \xi_{5} \Delta \xi_{5}-\xi_{0} \xi_{3} \xi_{5}$ \\
\hline \hline
\end{tabular}

it can be shown that such operators satisfy eqs.(3), where the super-Poincaré algebra is derived from a generalization of the Chevalley product, between even subspaces of a Clifford algebra, pure spinor formalism. Using the triality principle framework and the pure spinor formalism the super-Poincaré algebra is derived, which is shown to have a deep geometrical structure behind.
[1] J. Wess and B. Zumino, Nuc. Phys. B70, 39 (1974).

[2] I. Benn and R. Tucker, An Introd. to Spinors and Geometry with appl. in Physics, Adam Hilger, Bristol 1987.

[3] É. Cartan, Leçons sur la Theorie des Spineurs, Hermann, Paris 1937.

[4] C. Chevalley, The algebraic theory of spinors, Columbia Univ. Press, New York 1954.

[5] M. A. De Andrade, M. Rojas, and F. Toppan, Int. J. Mod. Phys.
A16 4453 (2001) [hep-th/ 0005035$].$

[6] A. Crumeyrolle, Orthogonal and Symplectic Clifford Algebras: Spinor Structures, Kluwer, Dordrecht 1990.

[7] P. Budinich and A. Trautman, The Spinorial Chessboard, Springer-Verlag, Berlin 1989.

[8] P. Budinich, Found. Phys. 321347 (2002). 